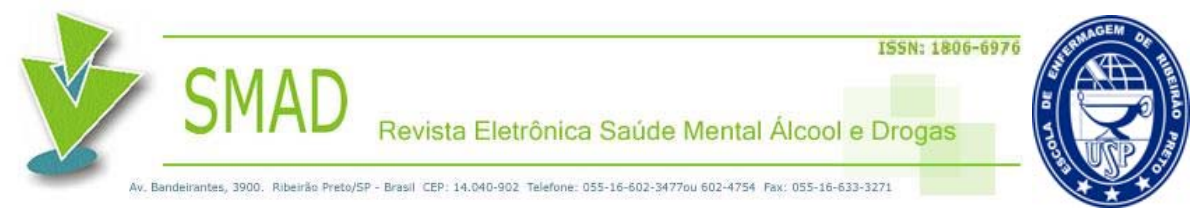

\title{
ALCOOLISMO E COMORBIDADE EM UM PROGRAMA DE ASSISTÊNCIA AOS DEPENDENTES DE ÁLCOOL
}

Flávia Batista Portugal ${ }^{1}$; Anna Paula Machado Corrêa ${ }^{2}$; Marluce Miguel de Siqueira $^{3}$

Resumo

O presente trabalho teve como objetivo descrever o perfil de pacientes atendidos no Programa de Atendimento ao Alcoolista do Hospital Universitário Cassiano Antônio Moraes da Universidade Federal do Espírito Santo (PAA-HUCAM-UFES), portadores de comorbidades. O estudo é descritivo, de caráter retrospectivo. Observa-se que a maioria dos pacientes atendidos no PAA são homens, na faixa etária entre 35 e 54 anos. A ocorrência de transtornos clínicos foi mais frequente, seguida dos transtornos psiquiátricos e sociais. Conhecer o perfil de uma população é de suma importância, pois conhecendo a situação é possível sugerir e programar ações para melhor atendimento.

Palavras-chave: Alcoolismo, Comorbidade, Tratamento.

ALCOHOLISM AND COMORBIDITY IN AN ALCOHOL DEPENDENCE THERAPEUTICAL PROGRAM

Abstract

This retrospective and descriptive study aimed to describe the profile of patients with comorbidity who receive care at the "Programa de Atendimento ao Alcoolista" of "Hospital Universitário Cassiano Antônio Moraes” from "Universidade Federal do Espírito Santo" (PAA-HUCAM-UFES). Most patients are male and were between 35 and 54 years of age. The occurrence of clinical disorders was the most frequent, followed by psychiatric and social disorders. It is very important to know the profile of a population, as thus it is possible to suggest and plan actions for a better care.

Keywords: Alcoholism, Comorbidity, Treatment.

ALCOHOLISMO Y COMORBILIDAD EN UN PROGRAMA DE ASISTENCIA AL DEPENDIENTES DEL ALCOHOL

Resumen

Este estudio descriptivo y retrospectivo tuvo como objetivo describir el perfil de pacientes con comorbilidades tratados en el "Programa de Atendimento ao Alcoolista" del "Hospital Universitário Cassiano Antônio Moraes" de la "Universidade Federal do Espírito Santo" (PAA-HUCAM-UFES). Se observa que la mayoría de los pacientes tratados en el PAA son hombres, con edad entre 35 y 54 años. La ocurrencia de los trastornos clínicos fue más frecuente, seguido por los psiquiátricos y sociales. Conocer el perfil de una población es muy importante, porque el conocimiento de la situación permite sugerir y planificar acciones para una mejor atención.

Palabras clave: Alcoholismo, Comorbilidad, Tratamiento.

\footnotetext{
${ }^{1}$ Enfermeira, Mestranda em Saúde Coletiva PPGSC-UFES e membro da Equipe Técnica do NEAD-CCS-UFES, e-mail flaviabportugal@gmail.com

${ }^{2}$ Enfermeira.e-mail paulinha_mc@hotmail.com

${ }^{3}$ Professora Associada II do Depto de Enfermagem, Coordenadora Científica do NEAD-CCS- Universidade Federal do Espírito Santo, Orientadora.e-mail marluce@ccs.ufes.br

SMAD $2010 \begin{aligned} & \begin{array}{l}\text { Volume } \\ \text { Volumen }\end{array} \\ & \text { S } 6\end{aligned} \begin{aligned} & \begin{array}{l}\text { Número } \\ \text { Numero } \\ \text { Number }\end{array} \\ & 1\end{aligned} \begin{aligned} & \begin{array}{l}\text { Artigo } \\ \text { Artículo } \\ \text { Article }\end{array} 05 \\ & \text { www.eerp.usp.br/resmad }\end{aligned}$
} 


\section{INTRODUÇÃO}

O uso do álcool é muito antigo, alguns historiadores acreditam que a data aproximada seria de 6.000 a.C. ${ }^{(1)}$. Atualmente, o consumo de álcool atingiu proporções espetaculares. A Organização Mundial de Saúde (OMS) estima que 2 bilhões de pessoas consumam bebidas alcoólicas e 76,3 milhões possuem o diagnóstico de transtornos, decorrente do seu consumo, além de ser o responsável por 1.8 milhões de mortes anualmente ${ }^{(2)}$.

O uso cada vez maior e os danos provocados, direta ou indiretamente, pelo consumo do álcool resulta na presença cada vez maior de comorbidades. Assim, “a ocorrência de uma patologia qualquer em um indivíduo já portador de outra doença, com potencialização entre essas, é conhecida como comorbidade”(3).

Os problemas relacionados ao uso de álcool e a sua associação com transtornos psiquiátricos são comuns. Os transtornos do humor, como a depressão, os transtornos de ansiedade, os transtornos de conduta, o déficit de atenção e hiperatividade, a esquizofrenia e o tabagismo são as comorbidades mais comuns associadas ao abuso de substâncias psicoativas $(\mathrm{SPAs})^{(4)}$.

Já entre as comorbidades físicas, o fígado é o principal órgão afetado pelo álcool. Existem basicamente três tipos de doenças do fígado determinadas pelo uso de álcool: esteatose (fígado gorduroso), hepatite alcoólica e cirrose alcoólica. Além do fígado, o álcool pode afetar outros órgãos do sistema gastrointestinal, tais como o pâncreas e o estômago. No pâncreas, o álcool é um dos principais fatores responsáveis pela pancreatite aguda e crônica ${ }^{(5-}$ 6).

Além dos danos físicos e psíquicos, observa-se crescimento do consumo de álcool paralelo ao aumento dos problemas sociais. Problema no trabalho é uma das principais consequências sociais do consumo abusivo de álcool. O consumo elevado gera baixa

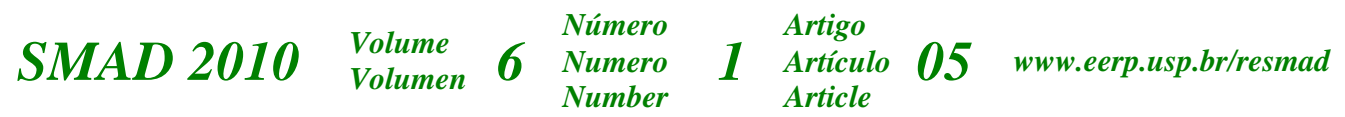


produtividade, aumento de lesões ao indivíduo e a terceiros, absenteísmo (além de impontualidade para chegar ao trabalho), suspensões disciplinares e problemas de relacionamento $^{(4,7)}$. O relacionamento familiar de alguém com consumo de álcool e seus familiares é complexo. Cônjuges e filhos são os que mais sofrem com as consequências da situação. Estudos demonstram estreita relação entre o consumo excessivo e o risco de crises matrimoniais, outros estudos mostram a existência de risco muito maior de separação entre bebedores intensos, em comparação a outras pesquisas ${ }^{(7)}$. Além desses, outro problema, frequentemente associado ao beber, é o dirigir alcoolizado. Assim, “estima-se que de 4 a 87\% (dependendo da amostra e jurisdição) dos motoristas alcoolizados podem ser considerados “alcoolistas” ou “bebedores problema” $[\ldots]^{(6)}$.

Estudos desenvolvidos no Programa de Atendimento ao Alcoolista (PAA) demonstram a associação entre alcoolismo e comorbidade tais como tabagismo ${ }^{(8)}$, depressão ${ }^{(9)}$, hipertensão ${ }^{(10)}$. Desse modo, traçar perfil das comorbidades nos pacientes que frequentaram o PAA contribui para o planejamento de tratamento diferenciado, para que o mesmo seja eficaz e eficiente para os alcoolistas portadores de comorbidades.

\section{MATERIAL E MÉTODOS}

O estudo foi realizado no Programa de Atendimento ao Alcoolista (PAA), localizado no Ambulatório de Clínica Médica do Hospital Universitário Cassiano Antônio de Moraes (HUCAM) da Universidade Federal do Espírito Santo (UFES). Trata-se de estudo descritivo, de caráter retrospectivo, no qual foi utilizada a pesquisa documental nos prontuários de todos os pacientes que frequentaram o PAA-HUCAM-UFES, no período da investigação.

A pesquisa foi realizada no período de abril a junho de 2008, no qual foram atendidos 240 pacientes. No entanto, 65 prontuários não foram encontrados pelo Serviço de Arquivo SMAD $2010 \begin{aligned} & \text { Volume } \\ & \text { Volumen }\end{aligned} 6 \begin{aligned} & \text { Número } \\ & \text { Numero } \\ & \text { Number }\end{aligned} \quad 1 \quad \begin{aligned} & \text { Artigo } \\ & \text { Artículo } \\ & \text { Article }\end{aligned} 05$ www.eerp.usp.br/resmad 
Médico Estatístico (SAME) do HUCAM, devido a problemas com dados de identificação. Além disso, foram excluídos 3 prontuários por não apresentarem diagnóstico prévio de alcoolismo, e 12 por terem sido atendidos por um profissional, o que resultaria em questionários com informações insuficientes. Desse modo, o estudo ficou composto por amostra de 160 pacientes acompanhados no PAA, nas consultas de primeira vez e de retorno.

Os dados foram coletados pelos pesquisadores após a assinatura do termo de consentimento institucional. Utilizando-se, para isso, um questionário, previamente testado, com questões do tipo fechadas e abertas, composto por três partes: I. dados sociodemográficos, II. história clínica do alcoolismo, sendo essa subdividida em: seguimento no PAA - enfermagem, medicina, psicologia e serviço social e III. comorbidades.

Os dados foram tabulados e analisados através do programa estatístico Statistical Package for the Social Science (SPSS) versão 15.0, sendo efetuada uma análise descritiva.

O estudo foi aprovado pelo Comitê de Ética em Pesquisa do Centro de Ciências da Saúde da Universidade Federal do Espírito Santo (Proc. $\mathrm{n}^{0} .108 / 08$ ), sendo desenvolvido de acordo com o estabelecido na Resolução $\mathrm{n}^{0}$.196, de 10.10.96, do Conselho Nacional de Saúde.

\section{RESULTADOS}

Após análise de 160 prontuários de pacientes atendidos no PAA, observa-se, na Tabela 1, que $86,9 \%$ eram do sexo masculino, sendo que a relação de homem para mulher era de 6,6:1. Quanto à faixa etária, verificou-se maior incidência entre 45 e 54 anos (35,7\%), com média de idade de 48,47 $( \pm 10,94)$ e idade mínima 22 anos e máxima 78. A maioria dos pacientes possuía o ensino fundamental completo e incompleto (42,3\%), seguido por 34\% de analfabetos. 50,6\% encontravam-se em união estável, enquanto 25,3\% eram separados.

SMAD $2010 \begin{aligned} & \text { Volume } \\ & \text { Volumen }\end{aligned} 6 \begin{aligned} & \text { Número } \\ & \text { Numero } \\ & \text { Number }\end{aligned} \quad 1 \quad \begin{aligned} & \text { Artigo } \\ & \text { Artículo } \\ & \text { Article }\end{aligned} 05$ www.eerp.usp.br/resmad 
Tabela 1 - Características sociodemográficas dos pacientes no PAA-HUCAM-UFES, 2008

\begin{tabular}{ccc}
\hline Características & n & \% \\
\hline Sexo & & \\
$\quad$ Masculino & 139 & 86,9 \\
Feminino & 21 & 13,1
\end{tabular}

Faixa etária

$\begin{array}{ccc}25 \text { a } 34 \text { anos } & 16 & 10,2 \\ 35 \text { a 44 anos } & 39 & 24,8 \\ 45 \text { a 54 anos } & 56 & 35,7 \\ 55 \text { a } 64 \text { anos } & 31 & 19,7 \\ \text { 65 a 74 anos } & 12 & 7,6 \\ \text { Acima de 75 anos } & 3 & 1,9 \\ \text { NR } & 3 & -\end{array}$

\section{Escolaridade}

$\begin{array}{ccc}\text { Analfabeto } & 33 & 34 \\ \text { EF } & 41 & 42,3 \\ \text { EM } & 21 & 21,6 \\ \text { ES } & 2 & 2,1 \\ \text { NR } & 63 & -\end{array}$

\section{Estado civil}

\begin{tabular}{ccc} 
Solteiro & 31 & 19,6 \\
União estável & 80 & 50,6 \\
Separado & 40 & 25,3 \\
Viúvo & 7 & 4,4 \\
NR & 2 & - \\
\hline Total & $\mathbf{1 6 0}$ & $\mathbf{1 0 0}$ \\
\hline
\end{tabular}

Entre os pacientes da pesquisa, 39,4\% experimentaram álcool na faixa etária de 15 a 19 anos, 31,3\% entre 10 e 14 anos e 10\% entre 20 e 24 anos. A idade mínima encontrada foi de 4 anos e máxima de 40, sendo a média de idade de 15,91, com desvio padrão de 5,43. Como mostrado na Tabela 2, o número de doses normalmente consumidas pelos pacientes, antes do início do seu tratamento, foi de 21 a 25 doses (26,3\%), 6 a 10 doses (23,4\%), 1 a 5 doses (19\%) e 11 a 15 doses (12,4\%). A maioria dos pacientes negou o uso de substâncias ilícitas (75,9\%). Sendo as SPA's mais encontradas, entre os que já usaram, a maconha

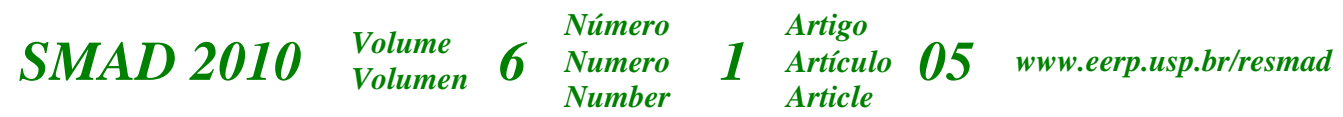


(67,9\%), cocaína $(57,1 \%)$, crack $(21,4 \%)$ e outras substâncias, tais como rebite e inalantes $(14,3 \%)$

Tabela 2 - Características do consumo de álcool dos pacientes no PAA-HUCAM-UFES, 2008

\begin{tabular}{ccc}
\hline Consumo de álcool & n & \% \\
\hline Quantidade em doses & & \\
1 a 5 doses & 26 & 19 \\
6 a 10 doses & 32 & 23,4 \\
11 a 15 doses & 17 & 12,4 \\
16 a 20 doses & 6 & 4,4 \\
21 a 25 doses & 36 & 26,3 \\
26 a 30 doses & 4 & 2,9 \\
31 a 35 doses & 2 & 1,5 \\
36 a 40 doses & 2 & 1,5 \\
Mais de 41 doses & 12 & 8,8 \\
NR & 23 & - \\
Bebidas mais consumidas & & \\
Cerveja & & \\
Cachaça & 44 & 31 \\
Vinho & 102 & 71,8 \\
Conhaque & 4 & 2,8 \\
Outros destilados & 6 & 4,2 \\
NR & 11 & 7,7 \\
& 18 & - \\
\hline
\end{tabular}

Observou-se maior ocorrência de transtornos clínicos, sendo diagnosticados em 113 pacientes, o que corresponde a 70,6\% de todos os pacientes. Posteriormente, encontraram-se os transtornos psiquiátricos (102-63,7\%) e sociais (55-34,4\%).

Como mostra a Tabela 3, entre os transtornos clínicos, a hipertensão foi o transtorno mais encontrado (33,1\%), seguido pelas doenças do aparelho gastrointestinal (30,6\%), pelo diabetes (8,8\%), doenças do aparelho respiratório (3,1\%) e parasitoses intestinais (2,5\%). Entre os transtornos do aparelho gastrointestinal, a esteatose prevaleceu (14 casos), seguida pela cirrose (12) e hepatite viral (11).

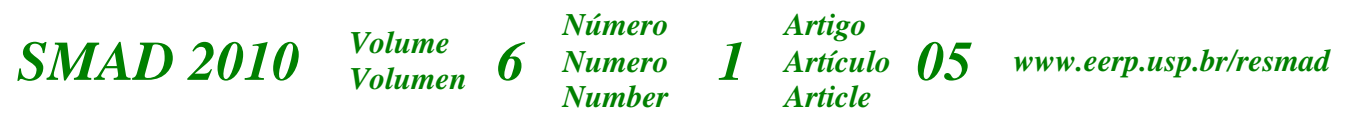


Tabela 3 - Comorbidades apresentadas pelos pacientes no PAA-HUCAM-UFES, 2008

\begin{tabular}{lcc}
\multicolumn{1}{c}{ Comorbidades } & $\mathbf{n}$ & $\mathbf{\%}$ \\
\hline Clínicas & & \\
Hipertensão & 53 & 33,1 \\
Doenças do aparelho gastrointestinal & 49 & 30,6 \\
Diabetes & 14 & 8,8 \\
Doenças do aparelho respiratório & 5 & 3,1 \\
Parasitoses intestinais & 4 & 2,5 \\
AVC/ICC & 3 & 1,9 \\
Epilepsia & 3 & 1,9 \\
Varizes de esôfago & 3 & 1,9 \\
Dislipidemia & 3 & 1,9 \\
Polineuropatia periférica & 2 & 1,3 \\
Impotência sexual & 2 & 1,3 \\
Wernick Korsackoff & 1 & 0,6 \\
Outros & 12 & 7,5 \\
Psiquiátricas & & \\
Tabagismo & & \\
Depressão & 93 & 58,1 \\
Uso de substâncias, exceto tabaco & 14 & 8,8 \\
Esquizofrenia & 4 & 2,5 \\
Outros & 3 & 1,9 \\
Problemas sociais & 3 & 1,9 \\
Conjugais & & \\
Laborais & 29 & 18,1 \\
Com filhos & 20 & 12,5 \\
Financeiros & 13 & 8,1 \\
Agressões & 7 & 4,4 \\
Habitacionais & 5 & 3,1 \\
Familiares & 4 & 2,5 \\
& 3 & 1,9 \\
\hline
\end{tabular}

Já entre as comorbidades psiquiátricas, o tabagismo foi o mais encontrado nos pacientes $(58,1 \%)$, seguido pela depressão $(8,8 \%)$. Entre os fatores sociais, $34,4 \%$ dos pacientes do PAA apresentaram algum problema. Dentre esses, os conjugais $(18,1 \%)$ e laborais (12,5\%) foram os mais prevalentes.

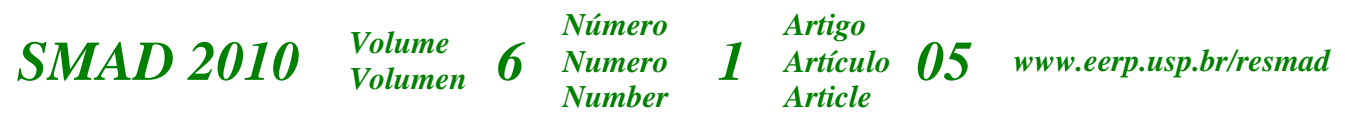




\section{DISCUSSÃO}

Na presente pesquisa, a maioria dos pacientes atendidos era do sexo masculino, condizente com o I Levantamento Nacional sobre os padrões de consumo de álcool na população brasileira, no qual a prevalência de bebedor pesado frequente foi de $9 \%$, sendo 14\% homens e $3 \%$ mulheres $^{(11)}$. Em outros estudos realizados no PAA, os índices foram semelhantes ao deste, sendo encontrado, em 2005: 91,4\% de homens e 8,6\% de mulheres ${ }^{(8)}$ e $88,2 \%$ e $11,8 \%{ }^{(12)}$ e em $2006: 90,2 \%$ e $9,8 \%{ }^{(9)}$; e $88,5 \%$ e $11,5 \%$, respectivamente ${ }^{(10)}$. Apesar de o alcoolismo ser mais encontrado em homens, as mulheres apresentam graves consequências do seu uso. Além disso, mulheres sofrem maior pressão social para parar o uso e, ainda, pesado julgamento social de tal ato, como também são mais suscetíveis às consequências para o desenvolvimento de complicações clínicas ${ }^{(13)}$. Já para a faixa etária, 60,5\% dos pacientes encontravam-se entre 35 e 54 anos. Estudos demonstram que esse é o grupo etário mais atingido pelo alcoolismo e, embora o consumo em geral comece cedo, os problemas relacionados ao seu uso ficam mais evidentes nessa faixa etária ${ }^{(14)}$.

No presente estudo, o número de doses normalmente consumido pelos pacientes do PAA, antes do início do seu tratamento, foi de 21 a 25 doses (26,3\%). Cinco, ou mais, doses para homens e 4, ou mais, para mulheres é considerado consumo de risco ${ }^{(11)}$, assim, os dados encontrados confirmam o fato de que a população atendida pelo PAA apresenta consumo de risco, uma vez que é constituída por pacientes dependentes de álcool. A bebida mais consumida pela população é a cerveja ${ }^{(15)}$, porém, entre os pacientes do PAA, a mais consumida é a cachaça (71,8\%). Esses pacientes possuem baixa escolaridade e baixa remuneração ${ }^{(16)}$, desse modo, a cachaça, por ser bebida de baixo custo, tende a ser a mais consumida.

SMAD $2010 \begin{aligned} & \text { Volume } \\ & \text { Volumen }\end{aligned} 6 \begin{aligned} & \text { Número } \\ & \text { Numero } \\ & \text { Number }\end{aligned} \quad 1$ Artigo $\begin{aligned} & \text { Artículo } \\ & \text { Article }\end{aligned} 05$ www.eerp.usp.br/resmad 
Já entre as comorbidades, os transtornos clínicos foram os mais frequentes. O predomínio de complicações clínicas deve-se ao fato de a população desse programa ser composta principalmente por faixa etária (35 e 54 anos), na qual os problemas decorrentes do consumo de álcool costumam aparecer ${ }^{(14)}$. A hipertensão foi a mais prevalente entre as complicações clínicas, em estudo realizado no $\mathrm{PAA}^{(10)}$, mostrando que 41,2\% eram hipertensos, dados semelhantes aos encontrados por este estudo. Tendo em vista que a hipertensão é acometimento de natureza multifatorial, acredita-se que o consumo de álcool é o segundo fator de risco não genético para a mesma ${ }^{(6)}$. Já entre as doenças do aparelho gastrointestinal, a esteatose foi a mais encontrada, uma vez que essa é a hepatopatia mais frequente, apesar de possível regressão do quadro, sua presença é importante, uma vez que, evoluindo, pode gerar cirrose hepática ${ }^{(17)}$.

Dos 102 pacientes que apresentaram uma, ou mais, comorbidade psiquiátrica, 97 apresentaram trasntorno relacionado ao uso de SPAs, o que corresponde a 60,6\% de todos os pacientes da pesquisa. Entre os pacientes que apresentram uso de SPAs, 93 usam o tabaco, o que corresponde à associação de alcoolismo e tabagismo de 58,1\% entre os pacientes deste estudo, como já encontrado em outros estudos realizados no $\mathrm{PAA}^{(8,12)}$. Várias pesquisas têm demonstrado essa associação, alguns, por exemplo, ressaltam que os tabagistas consumem duas vezes mais álcool do que os não tabagistas e que bebedores pesados tendem a estar associados ao tabagismo pesado. Há autores que explicam essa associação como forma de a nicotina antagonizar os efeitos depressores do álcool no sistema nervoso central ${ }^{(18)}$.

A depressão, posteriormente, foi a mais encontrada, embora, tanto o alcoolismo como a depressão sejam encontrados associados a outros transtornos mentais, as maiores taxas de comorbidades são encontradas na associação desses dois transtornos ${ }^{(19)}$. Acredita-se que os dependentes de álcool possuem dois ou três vezes mais chances para sofrer de transtornos

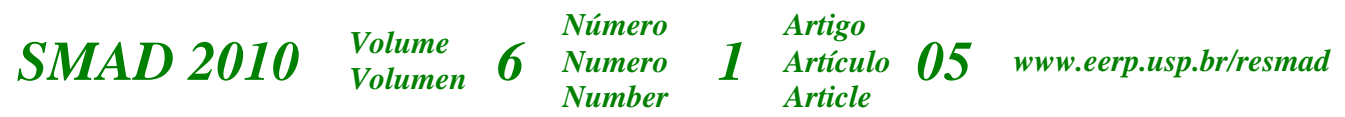


depressivos $^{(7)}$. Assim, em estudo realizado no PAA, encontrou-se taxa de $14,4 \%$ no PAA $^{(9)}$ e, em estudo realizado em São Paulo foi encontrado índice de 7,8\% de depressão grave, dados semelhantes aos encontrados por este estudo $(8,8 \%)^{(20)}$.

Como já mencionado, 34,4\% dos pacientes do PAA apresentaram problemas sociais. Estudos demonstram que bebedores intensos possuem risco maior para separação ou divórcio, além de aumentar o risco de violência conjugal. Ademais, o uso de álcool é uma das principais causas para a diminuição do rendimento no trabalho ${ }^{(7)}$.

\section{CONCLUSÃO}

Conhecer o perfil de uma população é de suma importância, já que assim é possível sugerir e programar ações para melhor atendimento. Desse modo, conhecer o perfil dos pacientes permite estabelecer ações para assistência eficaz ao paciente alcoolista.

Como já descrito na literatura, a faixa etária mais acometida foi de 35 a 54 anos, sendo a maioria dos pacientes do sexo masculino. Apesar de essa tendência ser encontrada constantemente, deve-se estar atento ao aumento de jovens alcoolistas. A incitação da mídia para o consumo de bebidas alcoólicas, em especial a cerveja, leva ao consumo cada vez mais precoce, o que, ao longo do tempo, pode gerar dependência.

Sendo assim, ações no âmbito geral, em nível governamental, são importantes. Contudo, deve-se apontar ações locais específicas para as peculiaridades dos pacientes. Apesar de os pacientes do PAA serem do sexo masculino, as mulheres representam um grupo importante, não pela sua expressão quantitativa, mas sim por suas especificidades. O alcoolismo como entidade clínica complexa, envolvendo vários aspectos da vida do ser humano, não pode ser resumido ao tratamento farmacológico. Assim, locais específicos de acompanhamento para as mulheres é suporte importantíssimo ao seu tratamento, auxiliando

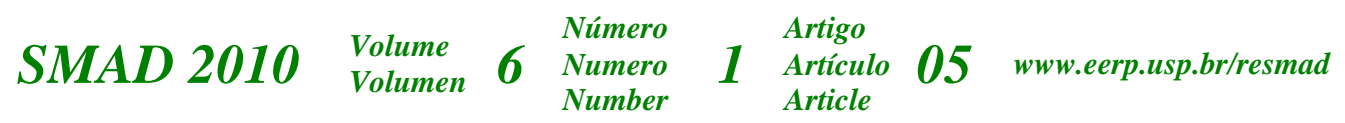


na manutenção da abstinência. Desse modo, é de grande relevância o relato desse fato no prontuário, para que, assim, todos os profissionais responsáveis pela paciente saibam do andamento e, possam estimular as pacientes a participarem de outras formas de tratamento, como, por exemplo, terapia em grupo.

Encontrou-se grande número de comorbidades clínicas em relação às outras comorbidades listadas no estudo. Fato explicável pela especialidade - todos os médicos do PAA são gastroenterologistas, logo, esses podem diagnosticar com mais precisão transtornos clínicos. Possivelmente, com a inserção de um psiquiatra no programa, poderia ser evidenciado o surgimento de transtornos psiquiátricos, os quais podem, muitas vezes, não serem diagnosticados por outros profissionais.

É importante salientar a necessidade de registro. Durante o estudo, foram notadas várias vezes a ausência da anotação de todos os profissionais. O registro mostra o que foi feito pelo profissional, além de fornecer informações importantes para a continuidade do tratamento.

\section{REFERÊNCIAS}

1-Centro Brasileiro de Informações sobre Drogas Psicotrópicas. O que são drogas psicotrópicas - Bebidas Alcoólicas: álcool etílico: etanol. Folheto Informativo. São Paulo: CEBRID; 2006.

2- WHO. Global Status Report on Alcohol. Geneva. Department of Mental Health and Substance Abuse.[cited 2008 June 01]. 2004. Available from:

http://www.who.int/substance_abuse/publications/alcohol/en/index.html

3- Ratto L, Cordeiro DC. Principais Comorbidades Psiquiátricas na Dependência química. In: Figlie NB, Bordin S, Laranjeira R. Aconselhamento em Dependência Química. São Paulo: Roca; 2004. p.167-86.

4- SENAD. Sistema para detecção do Uso abusivo e dependência de substâncias Psicoativas: Encaminhamento, intervenção breve, Reinserção social e Acompanhamento. São Paulo; 2006.

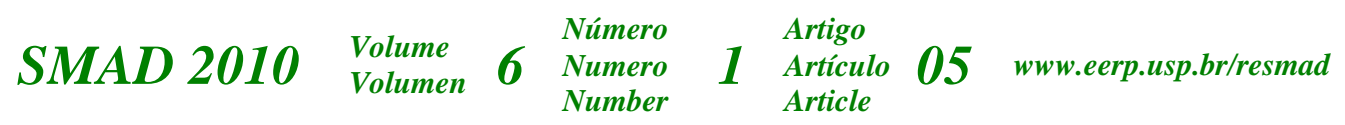


5- Figlie NB, Bordin S, Laranjeira R. Aconselhamento em Dependência Química. São Paulo: Roca; 2004.

6- Edwards G, Marshall EJ, Cook CCH. O Tratamento do Alcoolismo: Um Guia para Profissionais da Saúde. Porto Alegre: Artmed; 2005.

7- Anderson P, Gual A, Colon J. Alcohol y atención primaria de la salud: informaciones clínicas básicas para la identificación y el manejo de riesgos y problemas. Washington, D.C.: OPS; 2008.

8- Bossato JP. A Co-Dependência ao Álcool e à Nicotina em Pacientes do Programa de Atendimento ao Alcoolista da UFES. [Trabalho de Conclusão de Curso - Enfermagem e Obstetrícia]. Vitória: Departamento de Enfermagem/Universidade Federal do Espírito Santo; 2005.

9- Lempe AA, Stein JOS. Avaliação da Comorbidade Alcoolismo-Depressão no PAAHUCAM-UFES. [Trabalho de conclusão de curso]. Vitória: Departamento de Enfermagem/Universidade Federal do Espírito Santo; 2006.

10- Silva EP, Lima JM. Alcoolismo e Hipertensão: Impacto para a saúde. [Trabalho de conclusão de curso]. Vitória: Departamento de Enfermagem/Universidade Federal do Espírito Santo; 2006.

11- Laranjeira R, Illana P, Zaleski M, Caetano R. I Levantamento Nacional sobre os Padrões de Consumo de Álcool na População Brasileira. Brasília: Secretaria Nacional Antidrogas (SENAD); 2007.

12- Souza RS, Siqueira MM. O Processo de Enfermagem na Assistência a Pacientes com Dependência de Álcool. J Bras Psiquiatr. 2005; 54(3):228-33.

13- Nóbrega MPS, Oliveira EM. Mulheres usuárias de álcool: análise qualitativa. Rev Saúde Pública. outubro 2005; 39(5):816-23.

14- Lima MS. Epidemiologia do Alcoolismo. In: Ramos SP, Bertolote JM. Alcoolismo hoje. 3 ed. Porto Alegre: Artes Médicas; 1997. p.45-64.

15- Laranjeira R, Ribeiro MA Evolução do Conceito de Dependência Química. In: Gigliotti A, Guimarães A. Dependência, Compulsão e Impulsividade. Rio de Janeiro: Editora Rubio; 2007. p.9-17.

16- Garcia MLT, Macieira MS. Adesão ao tratamento entre alcoolistas. Arq Bras Psiquiatr Neurol Med Legal. janeiro-fevereiro-março 2003; 84-85:13-8.

SMAD $2010 \begin{aligned} & \text { Volume } \\ & \text { Volumen }\end{aligned} 6 \begin{aligned} & \text { Número } \\ & \text { Numero } \\ & \text { Number }\end{aligned} \quad 1$ Artigo $\begin{aligned} & \text { Artículo } \\ & \text { Article }\end{aligned} 05$ www.eerp.usp.br/resmad 
17- Matos LC. Doença Hepática Alcoólica (DHA). Med Interna. Viseu, julho-setembro 2006; 13(3):207-16.

18- Gigliotti A, Lemos T. Comorbidade Psiquiátrica em Tabagismo $\times$ Dependência de Álcool e outras substâncias. Rev ABEAD - Associação Brasileira de Estudo de Álcool e outras Drogas. Comorbidades: transtornos mentais x transtornos por uso de substâncias de abuso. São Paulo: ABEAD; 2005. p.55-60.

19- Abelardino V. Transtornos Depressivos $\times$ Dependência de Álcool e outras substâncias. Rev ABEAD - Associação Brasileira de Estudo de Álcool e outras Drogas. Comorbidades: transtornos mentais x transtornos por uso de substâncias de abuso. São Paulo: ABEAD; 2004. p.34-6.

20- Ratto LRC. Prevalência da Comorbidade entre Transtornos Mentais Graves e Transtornos Devido ao Uso de Substâncias Psicoativas em São Paulo, Brasil. 2000. [Dissertação Mestrado em Medicina]. São Paulo: Comissão de Pós-Graduação/FM/USP; 2000.

21- Morel CMA. Pesquisa em saúde e os objetivos do milênio: desafios e oportunidades globais, soluções e políticas nacionais. Ciênc Saúde Colet. 2004; 9(2):261-70.

Recebido em: 10/2009

Aprovado em: 01/2010

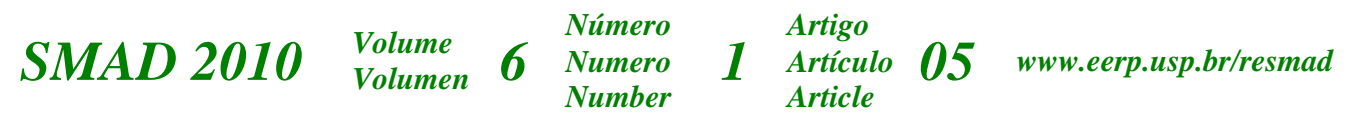

